\title{
Binding of Serotonin to the Human Serotonin Transporter. Molecular Modeling and Experimental Support
}

Leyla Celik, Steffen Sinning, Kasper Severinsen, Carsten Gram Hansen, Maria Sloth Møller, Mikael Bols, Ove Wiborg*, and Birgit Schiøtt*

\section{Materials and methods}

\section{Experimental Methods:}

Site-Directed Mutagenesis. Mutagenesis of hSERT cDNA in the pcDNA3 vector (Invitrogen) was carried out using complementary oligonucleotide primer pairs mismatched at the site of the desired point mutation in a polymerase reaction with Phusion High-Fidelity DNA Polymerase (Finnzymes). The polymerase reaction was digested for 12 hours with DpnI and used for transformation of supercompetent Solopack Gold (Stratagene) XL10 E. coli according to the manufacturers instructions. Colonies representing possible mutant clones were grown overnight at $37^{\circ} \mathrm{C}$ in LB medium (10 g/L Tryptone (Amresco), 5 g/L Yeast extract (Amresco), $10 \mathrm{~g} / \mathrm{L} \mathrm{NaCl}$ (Bie \& Berntsen), 200 ng/mL Ampicilin (Sigma), pH 7.0) in 96-well deepwell plates (Millipore) in a gyratory shaker. DNA was purified from these cultures using the Montage Plasmid Miniprep kit (Millipore) and subjected to sequencing on an ABI 3100 (Applied Biosystems) automated sequencer using BigDye Terminator v3.1 (Applied Biosystems) chemistry to identify the introduced mutation. Clones carrying the desired mutation were cultured in larger volumes and subjected to midiprep plasmid purification using the Nucleobond (Macherey-Nagel) or the PureYield (Promega) 
Plasmid midiprep kits. Full length sequencing of the hSERT cDNA gene in the mutant midiprep DNA was carried out to verify that no unwanted mutations had been introduced.

Uptake Assay. Two days prior to the uptake experiment cells were detached from the culture flask with trypsin/EDTA (BioWhitaker), transfected with midiprep DNA-Fugene6 (Roche) complex and seeded into white tissue culture treated 96-well microtiter plates (Nunc). Immediately before the uptake experiment was initiated, medium was aspirated and cells were washed once with PBSCM (137 mM NaCl, 27 mM KCl, 4.7 mM Na $2 \mathrm{HPO}_{4}, 1.2 \mathrm{mM} \mathrm{KH}_{2} \mathrm{PO}_{4}, 0.1 \mathrm{mM} \mathrm{CaCl} 2,1 \mathrm{mM}$ $\mathrm{MgCl}_{2}, 1 \mathrm{mM}$ ascorbic acid, $\mathrm{pH}$ 7.4). Cells were preincubated with a dilution of the drug in $40 \mu \mathrm{L}$ PBSCM for 30 minutes and uptake was initiated by the addition of $40 \mu \mathrm{L}$ of a dilution of the drug in PBSCM containing 50-100 nM [ $\left.{ }^{3} \mathrm{H}\right]-5-\mathrm{HT}$. Uptake was terminated after 10 minutes by aspiration and washing with PBSCM. All wash steps were done on a Bio-Tek Instruments ELX-50 automatic strip washer. $50 \mu \mathrm{L}$ of Microscint 20 (Packard) was dispensed into each well resulting in cell lysis and thereby release of accumulated radiolabeled substrate from the adherent cells allowing direct quantitation on a Packard Topcounter. 5-HT concentration in the uptake buffer was quantitated using a Packard 1900CA Liquid Scintillation Analyzer.

Data Analysis. Data from uptake inhibition experiments and $\mathrm{K}_{\mathrm{M}}-\mathrm{V}_{\max }$ experiments were fitted to a sigmoidal dose-response curve and a hyperbolic one-site binding curve, respectively, by nonlinear regression analysis using the built-in tools in Prism3 (Graphpad). IC I0 $_{50}$ values were transformed to $\mathrm{K}_{\mathrm{i}}$ values as described by Cheng and Prusoff. ${ }^{1}$

Organic Synthesis. Ligand analogues 1B, 2C, 3A, 3B, and 3C are synthesized according to the procedures described below.

(1H-indole-3-yl)-N-Methanamine. ${ }^{2,3}$

$216 \mathrm{mg}$ indole-3-aldehyde $(0.5 \mathrm{mmol})$ was dissolved in $7 \mathrm{ml}$ acetic acid followed by $3.5 \mathrm{ml}$ $\mathrm{MeNH}_{2}$ (35\% in water, $54 \mathrm{mmol}$ ) and cooled to $0^{\circ} \mathrm{C}$. To the solution was added $96 \mathrm{mg} \mathrm{NaBH}_{3} \mathrm{CN}$ 
(1.52mmol). The mixture was stirred over night. $10 \mathrm{ml}$ saturated $\mathrm{NaCl}$-solution was added to the solution. The aqueous layer was extracted with 3x15 ml mixture of $\mathrm{CHCl}_{3}-\mathrm{MeOH}(95 \%-5 \%)$. The combined organic layers were washed with brine and dried over magnesium sulphate and concentrated under reduced pressure. The resulting oil was dissolved in ethyl acetate and extracted using 1.0 M HCl and $\mathrm{NaOH}$. The organic phase was dried and concentrated to yield $13.5 \mathrm{mg}$ (17\%) of $1 \mathbf{B}$.

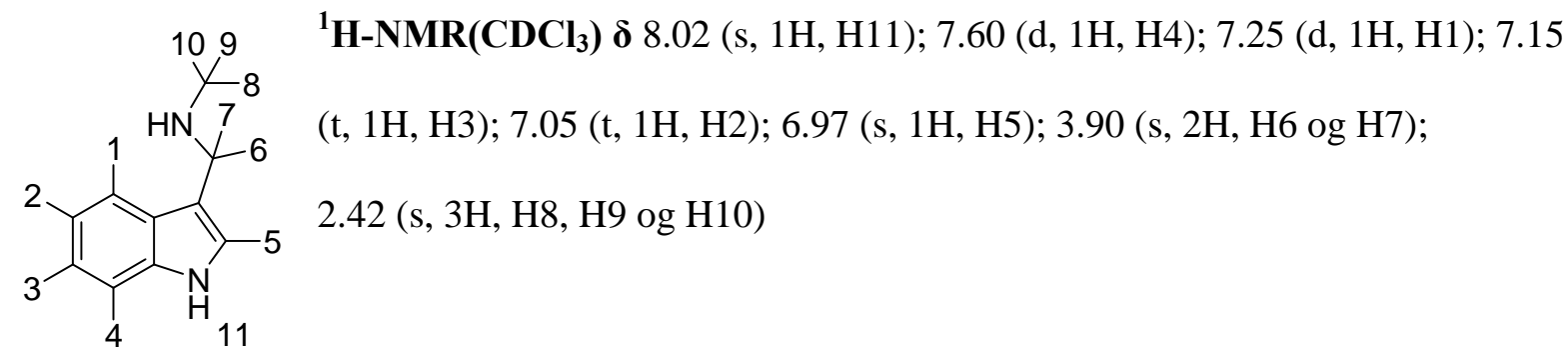

\section{2-(1H-indole-3-yl)-ethaneamine.,}

$290 \mathrm{mg}$ indole-3-aceticacid (2.7 mmol) was dissolved in $14.8 \mathrm{ml}$ dry THF followed by $348 \mathrm{mg}$ HOSu (4.0 mmol) and $518 \mathrm{mg}$ DCC (3.5 mmol). The mixture was stirred for two hours. To the mixture was then added $0.6 \mathrm{ml} \mathrm{Me}_{2} \mathrm{NH}$ (5 eq.). The mixture was stirred over night at room temperature. The mixture was filtrated, washed with $20 \mathrm{ml}$ DCM and concentrated to a yellow oil.

The oil was purified using flash chromatography $\left(\mathrm{CHCl}_{3}, 5 \% \mathrm{MeOH}, 1 \% \mathrm{Et}_{3} \mathrm{~N}\right)$ to yield $330 \mathrm{mg}$ (52\%) of the amide.

The amide was then dissolved in $1.6 \mathrm{ml}$ dioxane ${ }^{6}$ and it was transferred under an inert atmosphere to another flask containing $237 \mathrm{mg} \mathrm{LiAlH}_{4}$ (7 eq.) dissolved in $1.6 \mathrm{ml}$ dioxane. The reduction was carried out at $100^{\circ} \mathrm{C}$ over night. The reaction was quenched carefully with an aqueous solution of dioxane followed by washing with EtOAc. The solution was concentrated under reduced pressure to yield $250 \mathrm{mg}(46 \%)$ of $\mathbf{2 C}$. 

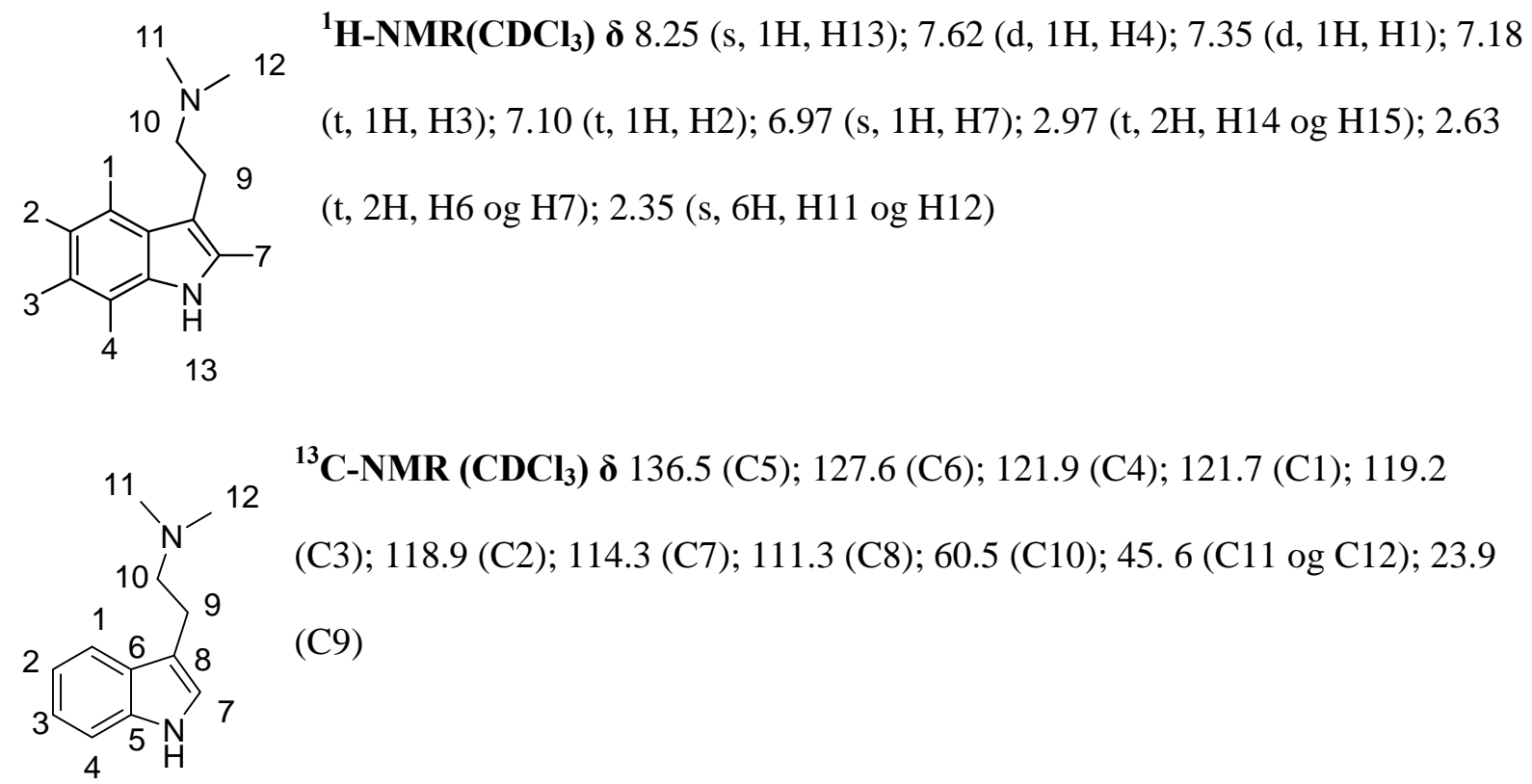

HR-MS (ES) Calculated for $\mathrm{C}_{12} \mathrm{H}_{16} \mathrm{~N}_{2}+\mathrm{Na}^{+}: \mathrm{m} / z$ 209.2132. Found : $m / z$ 209. 2021.

3-(1H-indole-3-yl)-propane-1-amine. ${ }^{7,8}(3 \mathrm{~A})$ :

$500 \mathrm{mg}$ indole-3-propanicacid (3.27 mmol) was dissolved in dry dioxane and $0.1 \mathrm{ml}$ pyridine (1.5 mmol) was added drop wise to the mixture. $605 \mathrm{mg}$ Boc-anhydride ( $3.4 \mathrm{mmol}$ ) was added. The reaction was stirred for 15 min and thereafter $\mathrm{NH}_{4} \mathrm{HCO}_{3}$ was added and the reaction stirred over night. $20 \mathrm{ml}$ EtOAc and 5\% $\mathrm{H}_{2} \mathrm{SO}_{4}$ was added to the mixture The organic phase was extracted with $3 x 15 \mathrm{ml}$ of water, dried over magnesium sulphate, and concentrated under reduced pressure to yield the amide, $228 \mathrm{mg}(45 \%)$.

The amide was then dissolved in $1.8 \mathrm{ml}$ dioxane and it was transferred under an inert

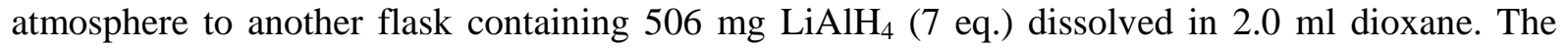
reduction was carried out at $100^{\circ} \mathrm{C}$ over night. The reaction was quenched carefully with an aqueous solution of dioxane followed by washing with EtOAc. The solution was concentrated under reduced pressure and purified using flash chromatography (80\%DCM, 5\%MeOH, $\left.1 \% \mathrm{Et}_{3} \mathrm{~N}\right)$ to yield $95 \mathrm{mg}(17 \%)$ of $\mathbf{3 A}$. 


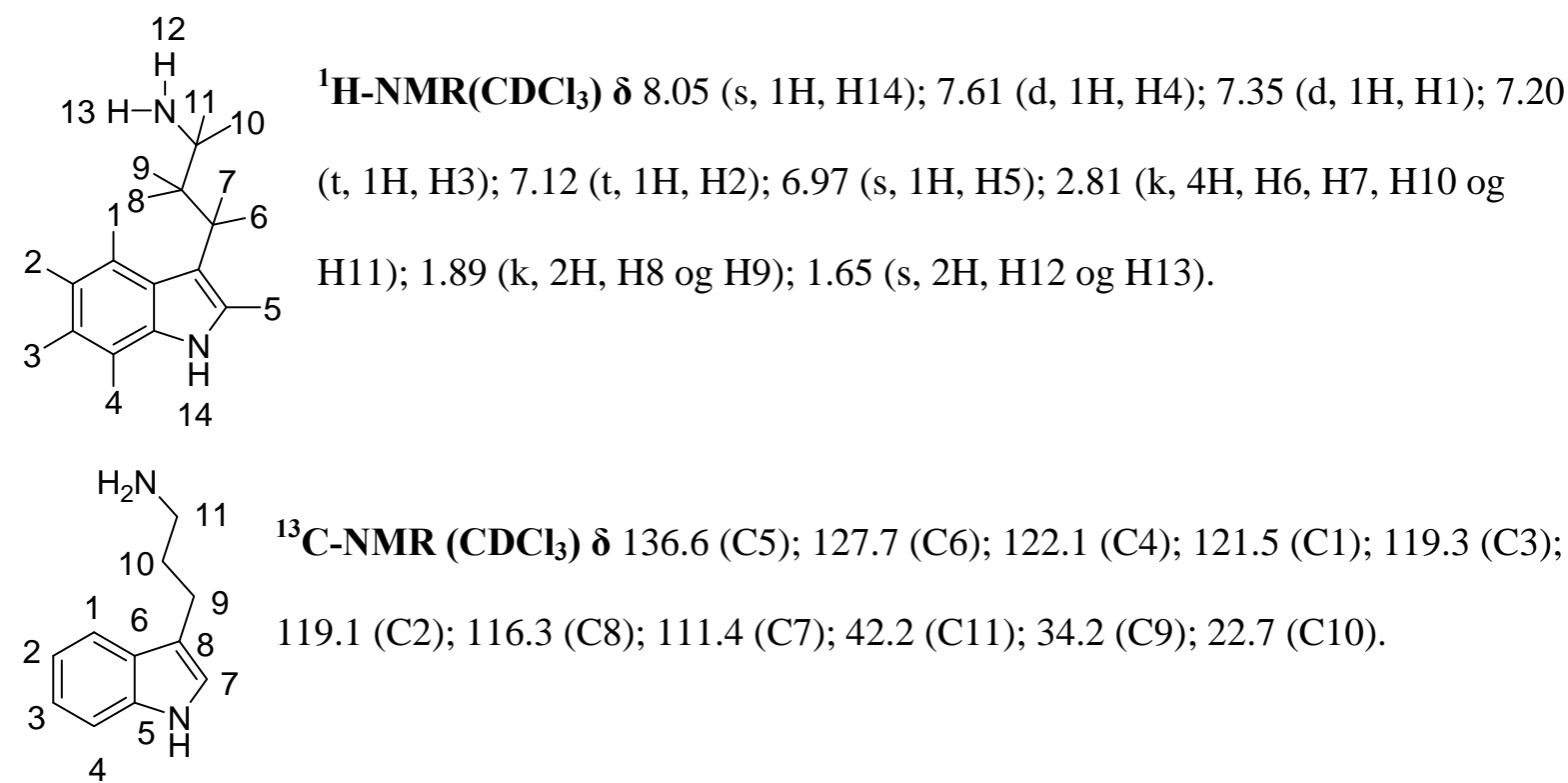

HR-MS (ES) Calculated for $\mathrm{C}_{11} \mathrm{H}_{13} \mathrm{~N}_{2}+\mathrm{Na}^{+}: \mathrm{m} / \mathrm{z}$ 175,1235. Found $m / z$ 175,1224.

\section{3-(1H-indole-3-yl)-N-Methylpropan-1-amine. ${ }^{9}$}

$500 \mathrm{mg}$ indole-3-propanicacid (2.7 mmol) was dissolved in $20 \mathrm{ml}$ dry THF followed by $456 \mathrm{mg}$ HOSu (4.0 mmol) and $700 \mathrm{mg}$ DCC (3.5 mmol). The mixture was stirred for two hours at room temperature. $0.6 \mathrm{ml}$ of $\mathrm{MeNH}_{2}$ (5 eq.) was then added to the mixture and the reaction left to stir over night at room temperature. The mixture was filtrated, washed with $20 \mathrm{ml}$ DCM and concentrated to a yellow oil.

The oil was purified using flash chromatography $\left(\mathrm{CHCl}_{3}, 5 \% \mathrm{MeOH}, 1 \% \mathrm{Et}_{3} \mathrm{~N}\right)$ to yield $116 \mathrm{mg}$ (23\%) of the amide.

The amide was then dissolved in $1.6 \mathrm{ml}$ dioxane and it was transferred under an inert

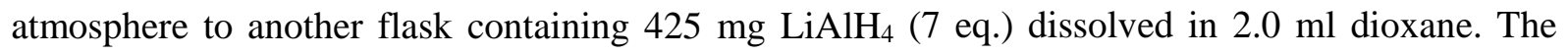
reduction was carried out at $100^{\circ} \mathrm{C}$ over night. The reaction was quenched with an aqueous solution 
of dioxane followed by washing with EtOAc. The solution was concentrated under reduced pressure and purified using flash chromatography to yield $18 \mathrm{mg}$ (4\%) of $\mathbf{3 B}$.

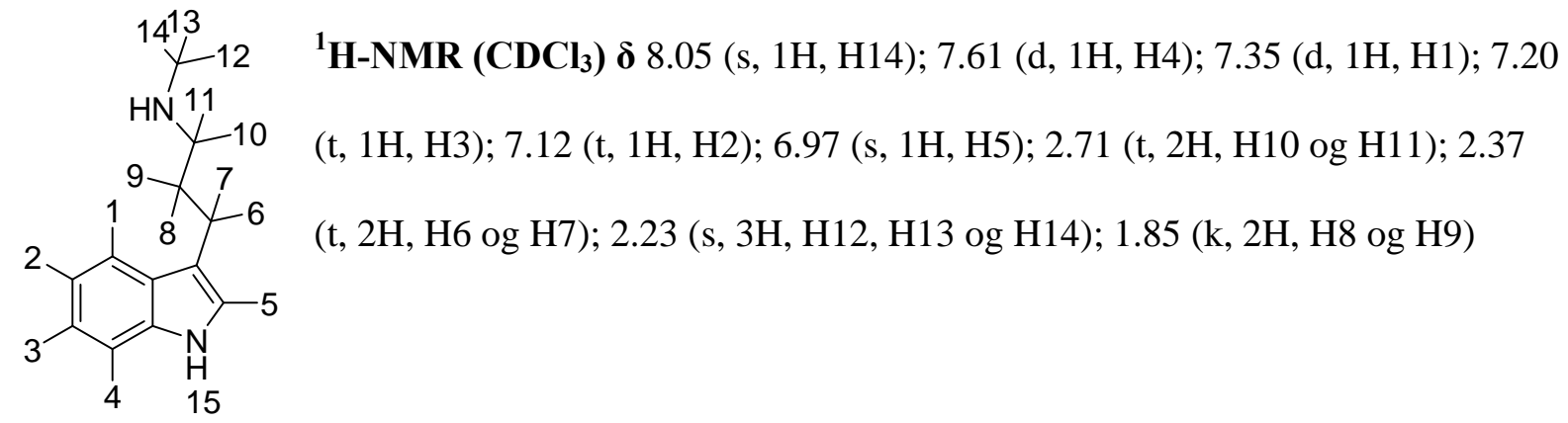

\section{3-(1H-indole-3-yl)-N-Diethylpropan-1-amine. ${ }^{10}$}

$500 \mathrm{mg}$ indole-3-propanicacid $(2.7 \mathrm{mmol}$ ) was dissolved in $20 \mathrm{ml}$ dry THF followed by $450 \mathrm{mg}$ HOSu (4.0 mmol) and $698 \mathrm{mg}$ DCC (3.5 mmol). The mixture was stirred for two hours at room temperature. $1.3 \mathrm{ml}$ of $\mathrm{MeNH}_{2}$ (5 eq.) was then added to the mixture. The reaction was stirred over night at room temperature. The mixture was filtrated, washed with $20 \mathrm{ml}$ DCM and concentrated to a yellow oil.

The oil was purified using flash chromatography $\left(\mathrm{CHCl}_{3}, 5 \% \mathrm{MeOH}, 1 \% \mathrm{Et}_{3} \mathrm{~N}\right)$ to yield $470 \mathrm{mg}$ (63\%) of the amide.

The amide was then dissolved in $1.6 \mathrm{ml}$ dioxane and it was transferred under an inert

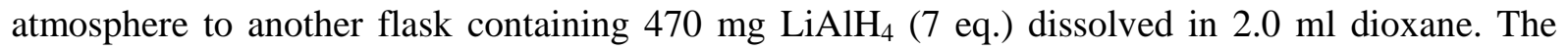
reduction was carried out at $100^{\circ} \mathrm{C}$ over night. The reaction was quenched carefully with an aqueous solution of dioxane followed by washing with EtOAc. The solution was concentrated under reduced pressure and purified using flash chromatography to yield $31 \mathrm{mg}$ (6\%) of $\mathbf{3 C}$. 


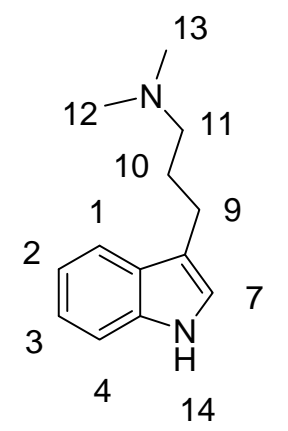

${ }^{1} \mathbf{H}-\mathbf{N M R}\left(\mathbf{C D C l}_{3}\right) \boldsymbol{\delta} 8.27$ (s, 1H, H14); 7.60 (d, 1H, H4); 7.37 (d, 1H, H1);

7.18 (t, 1H, H3); 7.10 (t, 1H, H2); 6.97 (s, 1H, H7); 2.79 (t, 2H, H11); 2.42

(t, 2H, H9); 2.27 (s, 6H, H12 og H13,); 1.96 (k, 2H, H10)

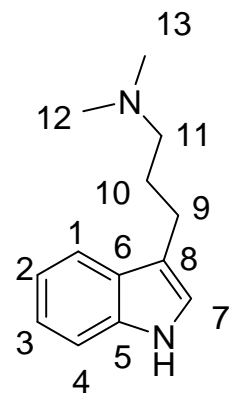

${ }^{13}$ C-NMR (CDCl $\left.\mathbf{3}\right) \boldsymbol{\delta} 136.6$ (C5); 127.7 (C6); 122.1 (4); 121.5 (1); 119.3 (C3);

119.1 (C2); 116.3 (C8); 111.3 (C7); 59.6 (C11); 45.4 (C12); 27.9 (C10); 23.1 (C9).

HR-MS (ES) Calculated for $\mathrm{C}_{13} \mathrm{H}_{18} \mathrm{~N}_{2}+\mathrm{Na}^{+}: \mathrm{m} / \mathrm{z}$ 203.1548. Found $m / z$ 203.1554. 


\section{Supplementary figures.}

Figure S1: The three employed alignments of hSERT against LeuT $\mathrm{T}_{A a}$. The darker blue the colour, the higher similarity there is between the alignments.

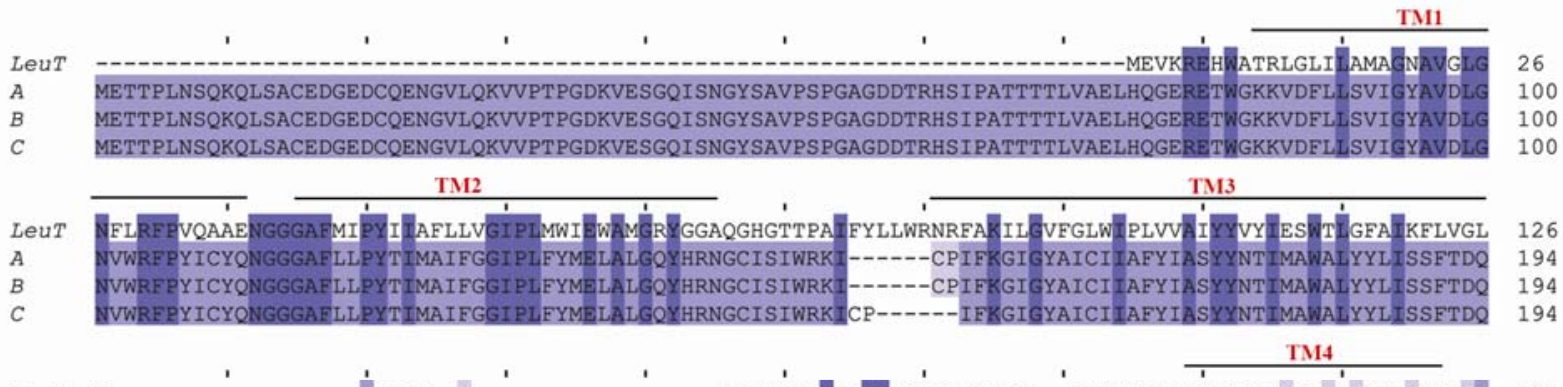

LeuT V---1--1'

A LPWTSCKNSWNTGNCTNYFSEDNI--T-----------WTLHSTSPAEEFYTRHVLQIHR---SKGLQDLGGISWQLALCIMLIFTVIYFS-I 270

B L---PWTSCKNSWNTGNCTNYFSED--NITWTLHSTSPAEEFYTRHVLQIHR---SKGLQDLG-GISWQLALCIMLIFTVIYFSI 270

C L---------PWTSC--KNSWNTGNCTNYFSEDNI TWTLHSTSPAEEFYTRHVLQIHRSKGLQDLGGISWQLALCIMLIFTVIYFSIWKGV 274

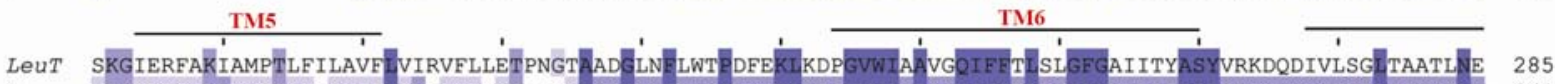
WKGVKTSGKVVWVTATFPYII SVLLVRGAT-LPGAWRGVLFYLKPNWQKLLETGVWIDAAAQIFESLGPGEGVLLAFASYNKFNNNCYQDALVTSVVNC 369 WKGVKTSGKVVWVTATFPYII SVLLVRGATIPG-AWRGVL FYLKPNWOKI KT-SGKVVWVTATFPYIILSVLLVRGATLP----GAWRGVLFYLKPNWOKLLETGVWIDAAAQIFESLGPGFGVLLAFASYNKFNNNCYQDALVTSVVNC 369

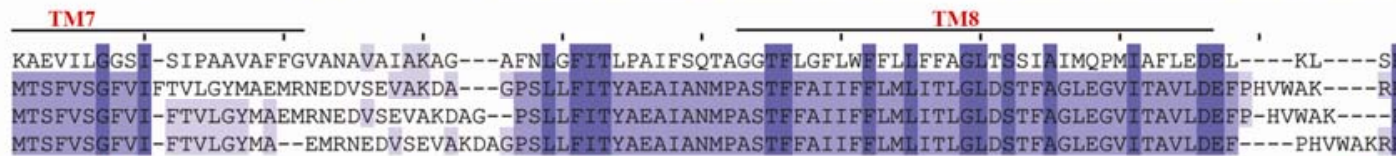

$$
\text { TM9 }
$$

TM10

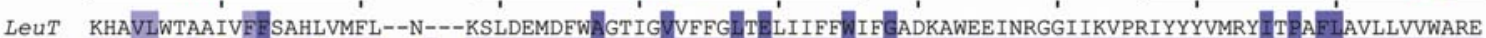
ERFVLAVVITCFEGSLVTLTF--GGA-YVVKLLEEYATGPAVLTVALIEAVAVSWFYGITQFCRDVKEMLGESPGWEWRICWVAISPLFLLFI ICSFLMS RERFVLAVVITCFFGSLVTLTF--GGAYVVKLLEEYATGPAVLTVALIEAVAVSWEYGITQFCRDVKEMLGFSPGWFWRICWVAISPLFLLFIICSFLM $\mathrm{TM} 12$

LEUT YIPKIMEETH---WT---VWITRFYIIGLËLFLTFLVFLA'ARRRNHESAGT

PPQLRLFOYN---YP--YWSI I LGYCIGTSSFICI PTYIAYRLI ITPGTFKERI IKS ITPETPTE I PCGDIRLNAV

PPQLRLFQYNY---PYWSI ILGYCI GTSSEICI PTYIAYRL I ITPGTFKERI IKSITPETPTE I PCGDIRLNAV

PPQLRLFQYNYPYWS---IILGYCIGTSSFICI PTY IAYRLI ITPGTFKERI I KS ITPETPTE I PCGDIRLNAV 
Figure S2: Computed DOPE-curves for alignments A, B, and C.
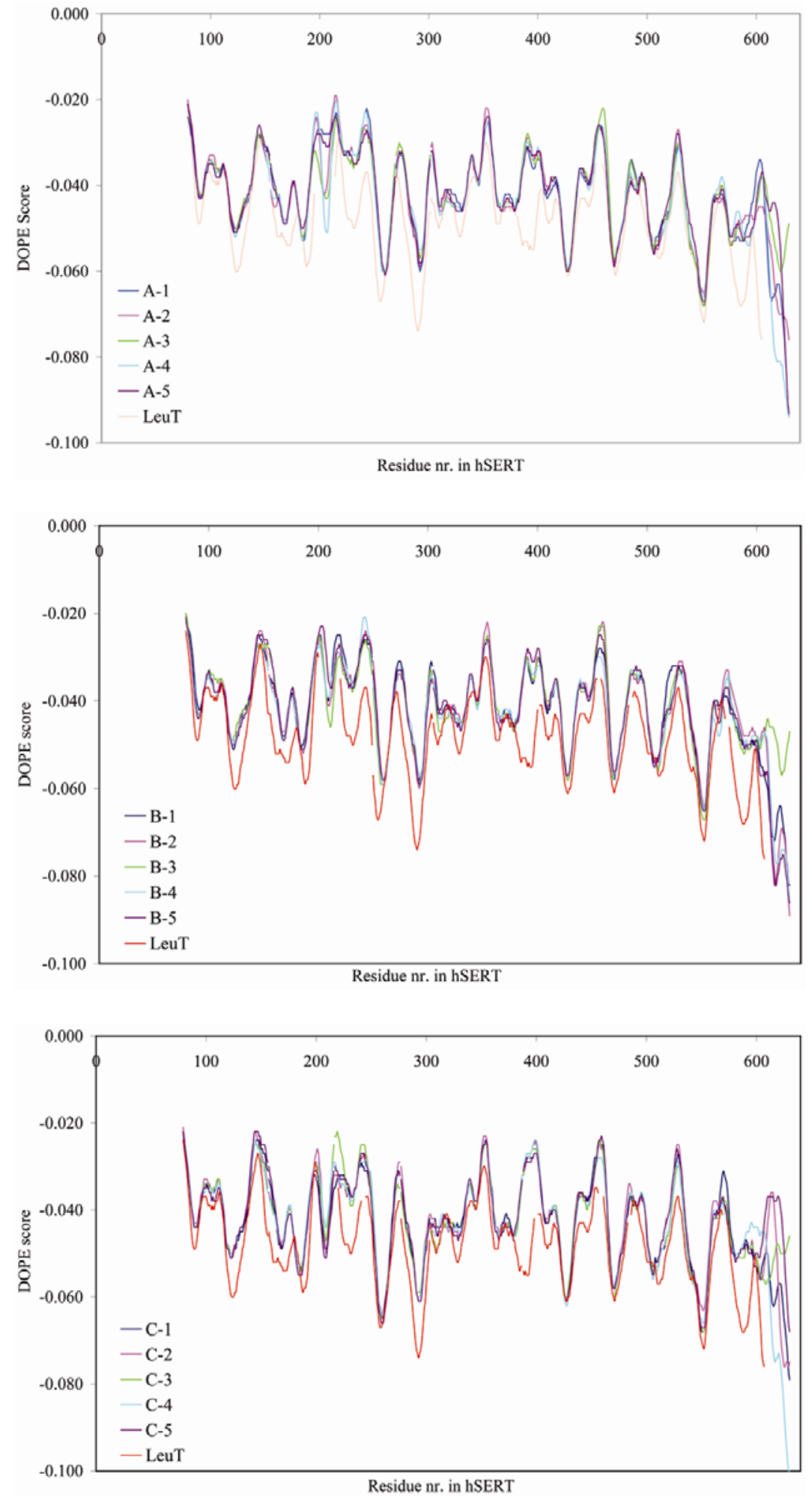
Table S1: Induced fit docking of 5-HT in the three selected homology models of hSERT. The setup number in column 1 refers to Table 2 in the manuscript; setups 1-6 correspond to alignment $\mathbf{A}$, setups 7-11 to alignment $\mathbf{B}$ and setups 12-18 to alignment $\mathbf{C}$. The poses are sorted with respect to cluster, IFDScore , and GlideScore.

\begin{tabular}{|c|c|c|c|c|c|c|c|}
\hline Setup & \begin{tabular}{|c} 
Prime E \\
(kcal/mol)
\end{tabular} & $\begin{array}{l}\text { IFDScore } \\
\text { (kcal/mol) }\end{array}$ & $\begin{array}{c}\text { GlideScore } \\
\text { (kcal/mol) }\end{array}$ & $\begin{array}{c}\text { E-Model } \\
\text { (kcal/mol) }\end{array}$ & Cluster & $\begin{array}{c}\text { Dist } 5-\mathrm{HT}(\mathrm{N}) \ldots \\
\text { D98(O1) }(\AA)\end{array}$ & $\begin{array}{c}\text { Dist 5-HT(N) )... } \\
\text { D98(O2) }(\AA)\end{array}$ \\
\hline 16 & -20539 & -1038.15 & -11.18 & -98.19 & $\mathrm{C} 1$ & 3.9 & 2.7 \\
\hline 16 & -20537 & -1038.04 & -11.18 & -99.00 & C1 & 4.0 & 2.9 \\
\hline 17 & -20533 & -1037.61 & -10.94 & -93.33 & C1 & 3.6 & 2.9 \\
\hline 17 & -20532 & -1037.60 & -11.00 & -97.53 & C1 & 4.0 & 2.9 \\
\hline 17 & -20523 & -1037.46 & -11.33 & -98.82 & $\mathrm{C} 1$ & 3.9 & 2.8 \\
\hline 17 & -20526 & -1037.38 & -11.10 & -96.93 & C1 & 3.9 & 2.8 \\
\hline 17 & -20522 & -1037.28 & -11.16 & -94.66 & C1 & 2.7 & 3.8 \\
\hline 17 & -20532 & -1037.05 & -10.45 & -93.20 & $\mathrm{C} 1$ & 4.5 & 2.6 \\
\hline 17 & -20527 & -1036.72 & -10.35 & -88.39 & $\mathrm{C} 1$ & 3.5 & 3.4 \\
\hline 16 & -20528 & -1036.72 & -10.31 & -94.58 & C1 & 4.5 & 2.5 \\
\hline 16 & -20526 & -1036.57 & -10.28 & -94.86 & $\mathrm{C1}$ & 4.5 & 2.5 \\
\hline 16 & -20529 & -1036.44 & -9.98 & -90.66 & $\mathrm{C} 1$ & 3.9 & 4.7 \\
\hline 17 & -20528 & -1036.35 & -9.98 & -94.05 & C1 & 4.5 & 2.6 \\
\hline 16 & -20516 & -1036.23 & -10.42 & -96.47 & $\mathrm{C1}$ & 4.5 & 2.6 \\
\hline 17 & -20521 & -1035.91 & -9.85 & -89.58 & $\mathrm{C} 1$ & 4.6 & 2.7 \\
\hline 17 & -20520 & -1035.58 & -9.59 & -94.70 & $\mathrm{C} 1$ & 4.5 & 2.6 \\
\hline 5 & -20477 & -1034.59 & -10.72 & -91.90 & $\mathrm{C1}$ & 3.6 & 2.9 \\
\hline 5 & -20488 & -1034.33 & -9.91 & -84.94 & $\mathrm{C} 1$ & 2.9 & 3.9 \\
\hline 5 & -20474 & -1033.75 & -10.03 & -89.71 & $\mathrm{C} 1$ & 3.4 & 4.5 \\
\hline 5 & -20476 & -1033.49 & -9.68 & -95.36 & $\mathrm{C1}$ & 2.7 & 3.9 \\
\hline 5 & -20464 & -1033.34 & -10.16 & -81.59 & $\mathrm{C} 1$ & 3.3 & 2.9 \\
\hline 5 & -20472 & -1033.14 & -9.54 & -84.74 & $\mathrm{C} 1$ & 2.9 & 3.7 \\
\hline 5 & -20489 & -1033.10 & -8.66 & -79.53 & $\mathrm{C} 1$ & 2.7 & 4.7 \\
\hline 18 & -19137 & -967.10 & -10.23 & -67.07 & $\mathrm{C} 1$ & 3.7 & 3.0 \\
\hline 18 & -19125 & -966.28 & -10.01 & -65.92 & $\mathrm{C} 1$ & 3.3 & 2.8 \\
\hline 15 & -19097 & -966.05 & -11.21 & -85.58 & $\mathrm{C} 1$ & 3.4 & 2.8 \\
\hline 18 & -19123 & -965.75 & -9.62 & -56.46 & $\mathrm{C} 1$ & 3.1 & 2.9 \\
\hline 15 & -19100 & -965.50 & -10.49 & -77.59 & $\mathrm{C} 1$ & 3.4 & 3.2 \\
\hline 18 & -19112 & -965.11 & -9.50 & -46.49 & $\mathrm{C} 1$ & 3.2 & 3.4 \\
\hline 18 & -19113 & -964.70 & -9.04 & -57.20 & $\mathrm{C} 1$ & 2.9 & 3.7 \\
\hline 15 & -19082 & -964.31 & -10.22 & -81.96 & $\mathrm{C} 1$ & 3.7 & 4.4 \\
\hline 15 & -19084 & -963.71 & -9.52 & -86.42 & $\mathrm{C} 1$ & 2.8 & 3.8 \\
\hline 15 & -19087 & -963.53 & -9.20 & -69.83 & $\mathrm{C} 1$ & 3.7 & 2.6 \\
\hline 15 & -19072 & -963.28 & -9.71 & -75.59 & $\mathrm{C} 1$ & 3.3 & 4.5 \\
\hline 14 & -18964 & -959.70 & -11.48 & -103.28 & $\mathrm{C} 1$ & 2.7 & 4.6 \\
\hline 14 & -18970 & -959.51 & -11.01 & -97.19 & $\mathrm{C} 1$ & 2.9 & 4.8 \\
\hline 14 & -18967 & -959.17 & -10.81 & -99.94 & $\mathrm{C} 1$ & 2.6 & 4.5 \\
\hline 14 & -18962 & -958.94 & -10.83 & -97.83 & $\mathrm{C} 1$ & 2.8 & 4.6 \\
\hline 14 & -18964 & -958.45 & -10.26 & -95.20 & $\mathrm{C} 1$ & 2.7 & 4.1 \\
\hline 14 & -18954 & -958.45 & -10.73 & -92.73 & $\mathrm{C} 1$ & 2.6 & 4.3 \\
\hline 14 & -18952 & -958.02 & -10.43 & -91.64 & $\mathrm{C} 1$ & 4.6 & 2.7 \\
\hline
\end{tabular}




\begin{tabular}{|c|c|c|c|c|c|c|c|}
\hline 13 & -18938 & -957.43 & -10.55 & -83.14 & C1 & 3.4 & 2.7 \\
\hline 13 & -18933 & -957.37 & -10.71 & -90.61 & $\mathrm{C} 1$ & 4.3 & 2.7 \\
\hline 13 & -18931 & -957.24 & -10.68 & -80.70 & $\mathrm{C} 1$ & 2.9 & 4.3 \\
\hline 13 & -18926 & -957.05 & -10.75 & -95.63 & $\mathrm{C} 1$ & 4.3 & 3.5 \\
\hline 13 & -18943 & -957.00 & -9.83 & -87.06 & C1 & 3.4 & 4.6 \\
\hline 13 & -18941 & -956.67 & -9.64 & -84.07 & C1 & 3.7 & 4.4 \\
\hline 13 & -18933 & -956.64 & -9.98 & -90.21 & $\mathrm{C} 1$ & 3.6 & 4.8 \\
\hline 6 & -18866 & -953.34 & -10.04 & -57.46 & C1 & 3.1 & 2.9 \\
\hline 4 & -18850 & -952.78 & -10.26 & -82.50 & C1 & 2.8 & 4.0 \\
\hline 4 & -18840 & -952.21 & -10.23 & -76.43 & $\mathrm{C} 1$ & 3.4 & 2.7 \\
\hline 4 & -18841 & -952.06 & -9.99 & -75.09 & $\mathrm{C} 1$ & 2.9 & 3.4 \\
\hline 6 & -18841 & -951.74 & -9.68 & -50.52 & $\mathrm{C} 1$ & 2.9 & 3.6 \\
\hline 6 & -18839 & -951.60 & -9.67 & -45.24 & $\mathrm{C} 1$ & 2.7 & 3.7 \\
\hline 4 & -18843 & -951.54 & -9.41 & -66.41 & $\mathrm{C} 1$ & 2.9 & 3.4 \\
\hline 4 & -18846 & -951.35 & -9.06 & -73.23 & $\mathrm{C} 1$ & 3.8 & 4.6 \\
\hline 6 & -18836 & -951.13 & -9.31 & -46.83 & $\mathrm{C} 1$ & 2.9 & 4.0 \\
\hline 12 & -18823 & -951.07 & -9.93 & -96.89 & C1 & 2.7 & 4.6 \\
\hline 12 & -18807 & -951.06 & -10.71 & -101.82 & C1 & 2.8 & 4.7 \\
\hline 4 & -18842 & -951.05 & -8.94 & -73.60 & C1 & 3.9 & 4.5 \\
\hline 12 & -18803 & -950.99 & -10.86 & -107.46 & C1 & 2.9 & 4.3 \\
\hline 12 & -18808 & -950.90 & -10.53 & -94.80 & C1 & 2.5 & 4.3 \\
\hline 12 & -18816 & -950.83 & -10.03 & -99.43 & C1 & 4.7 & 2.9 \\
\hline 12 & -18801 & -949.96 & -9.91 & -90.62 & $\mathrm{C} 1$ & 2.6 & 4.5 \\
\hline 12 & -18797 & -949.87 & -10.00 & -106.86 & C1 & 4.5 & 3.2 \\
\hline 3 & -18733 & -947.07 & -10.44 & -99.87 & $\mathrm{C} 1$ & 2.7 & 4.2 \\
\hline 3 & -18733 & -946.08 & -9.44 & -87.83 & $\mathrm{C} 1$ & 3.2 & 2.6 \\
\hline 3 & -18727 & -945.83 & -9.49 & -82.25 & $\mathrm{C} 1$ & 3.1 & 2.9 \\
\hline 3 & -18731 & -945.68 & -9.13 & -81.04 & C1 & 4.3 & 5.2 \\
\hline 2 & -18669 & -943.40 & -9.97 & -76.62 & $\mathrm{C} 1$ & 3.3 & 2.8 \\
\hline 2 & -18671 & -943.37 & -9.84 & -75.36 & $\mathrm{C} 1$ & 3.3 & 2.8 \\
\hline 2 & -18694 & -943.36 & -8.66 & -79.83 & $\mathrm{C} 1$ & 4.8 & 2.7 \\
\hline 2 & -18666 & -943.28 & -9.96 & -87.86 & $\mathrm{C} 1$ & 3.1 & 3.9 \\
\hline 2 & -18666 & -943.16 & -9.88 & -81.18 & $\mathrm{C} 1$ & 2.7 & 3.8 \\
\hline 1 & -18520 & -935.32 & -9.31 & -86.32 & $\mathrm{C} 1$ & 3.2 & 2.7 \\
\hline 1 & -18520 & -935.32 & -9.33 & -92.20 & $\mathrm{C} 1$ & 3.1 & 2.7 \\
\hline 5 & -20479 & -1033.19 & -9.23 & -74.29 & $\mathrm{C} 2$ & 3.6 & 2.7 \\
\hline 5 & -20474 & -1032.95 & -9.23 & -77.73 & $\mathrm{C} 2$ & 3.2 & 2.7 \\
\hline 15 & -19075 & -963.42 & -9.66 & -73.63 & C2 & 3.7 & 3.1 \\
\hline 4 & -18856 & -952.47 & -9.65 & -72.98 & $\mathrm{C} 2$ & 3.5 & 2.6 \\
\hline 4 & -18847 & -952.15 & -9.78 & -74.37 & $\mathrm{C} 2$ & 3.5 & 2.7 \\
\hline 6 & -18847 & -950.72 & -8.35 & -51.05 & $\mathrm{C} 2$ & 4.9 & 4.4 \\
\hline 4 & -18832 & -950.48 & -8.87 & -65.48 & $\mathrm{C} 2$ & 3.5 & 2.6 \\
\hline 6 & -18859 & -948.94 & -6.02 & -48.97 & $\mathrm{C} 2$ & 3.2 & 3.0 \\
\hline 3 & -18732 & -947.69 & -11.11 & -97.02 & $\mathrm{C} 2$ & 2.6 & 3.6 \\
\hline 2 & -18685 & -944.31 & -10.07 & -85.48 & $\mathrm{C} 2$ & 3.1 & 2.7 \\
\hline 2 & -18680 & -944.01 & -10.00 & -80.78 & $\mathrm{C} 2$ & 3.6 & 2.8 \\
\hline 2 & -18682 & -943.46 & -9.37 & -82.79 & $\mathrm{C} 2$ & 3.2 & 2.6 \\
\hline 2 & -18681 & -942.72 & -8.68 & -77.74 & $\mathrm{C} 2$ & 3.6 & 2.6 \\
\hline 1 & -18510 & -935.12 & -9.64 & -82.69 & $\mathrm{C} 2$ & 3.4 & 2.6 \\
\hline 1 & -18510 & -933.97 & -8.44 & -79.80 & $\mathrm{C} 2$ & 4.5 & 2.5 \\
\hline
\end{tabular}




\begin{tabular}{|c|c|c|c|c|c|c|c|}
\hline 16 & -20539 & -1036.99 & -10.06 & -88.10 & C3 & 2.9 & 4.7 \\
\hline 18 & -19116 & -966.32 & -10.54 & -62.70 & C3 & 3.9 & 2.7 \\
\hline 15 & -19086 & -963.64 & -9.33 & -79.10 & C3 & 4.7 & 3.7 \\
\hline 14 & -18953 & -958.33 & -10.66 & -102.75 & C3 & 2.7 & 4.1 \\
\hline 14 & -18951 & -957.62 & -10.05 & -93.98 & C3 & 4.1 & 2.6 \\
\hline 14 & -18949 & -957.47 & -10.02 & -95.16 & C3 & 2.7 & 4.3 \\
\hline 13 & -18939 & -956.47 & -9.54 & -84.50 & C3 & 5.1 & 3.8 \\
\hline 13 & -18939 & -956.17 & -9.24 & -81.91 & C3 & 4.0 & 5.0 \\
\hline 12 & -18808 & -951.91 & -11.50 & -112.26 & C3 & 2.6 & 4.1 \\
\hline 12 & -18794 & -950.34 & -10.61 & -108.23 & C3 & 4.1 & 2.6 \\
\hline 3 & -18726 & -948.18 & -11.87 & -89.39 & C3 & 2.7 & 4.1 \\
\hline 3 & -18730 & -947.07 & -10.57 & -83.60 & C3 & 2.7 & 4.1 \\
\hline 3 & -18726 & -945.73 & -9.42 & -71.40 & C3 & 2.9 & 4.4 \\
\hline 2 & -18684 & -943.14 & -8.96 & -86.35 & C3 & 3.0 & 3.3 \\
\hline 1 & -18533 & -935.71 & -9.06 & -86.28 & C3 & 2.7 & 3.8 \\
\hline 1 & -18509 & -934.61 & -9.15 & -91.53 & $\mathrm{C} 3$ & 2.7 & 3.9 \\
\hline 13 & -18921 & -955.11 & -9.04 & -75.67 & Outlier & 2.8 & 3.3 \\
\hline 6 & -18837 & -952.08 & -10.25 & -60.66 & Outlier & 2.6 & 3.1 \\
\hline 3 & -18715 & -946.95 & -11.21 & -93.44 & Outlier & 2.6 & 4.1 \\
\hline 7 & -18634 & -939.62 & -7.92 & -51.73 & Outlier & 2.8 & 4.3 \\
\hline 1 & -18520 & -935.99 & -9.99 & -96.81 & Outlier & 2.5 & 3.9 \\
\hline 11 & -18901 & -952.63 & -7.57 & -67.26 & Vestibule & 15.0 & 14.7 \\
\hline 10 & -18901 & -952.29 & -7.22 & -67.45 & Vestibule & 16.2 & 15.9 \\
\hline 8 & -18709 & -944.48 & -9.03 & -68.78 & Vestibule & 9.8 & 8.6 \\
\hline 9 & -18692 & -943.80 & -9.22 & -67.36 & Vestibule & 7.8 & 6.6 \\
\hline 9 & -18700 & -942.30 & -7.30 & -58.83 & Vestibule & 15.0 & 15.4 \\
\hline 8 & -18679 & -941.94 & -7.99 & -55.53 & Vestibule & 9.5 & 7.9 \\
\hline 9 & -18708 & -941.55 & -6.14 & -54.22 & Vestibule & 17.3 & 17.6 \\
\hline 9 & -18680 & -941.52 & -7.52 & -55.87 & Vestibule & 7.5 & 6.4 \\
\hline 8 & -18686 & -941.42 & -7.10 & -58.73 & Vestibule & 7.9 & 6.6 \\
\hline 9 & -18702 & -940.98 & -5.88 & -60.28 & Vestibule & 14.5 & 13.9 \\
\hline 9 & -18683 & -940.62 & -6.47 & -63.20 & Vestibule & 7.7 & 6.5 \\
\hline 7 & -18640 & -939.25 & -7.25 & -71.09 & Vestibule & 16.2 & 16.2 \\
\hline 7 & -18622 & -938.42 & -7.35 & -65.92 & Vestibule & 3.0 & 3.2 \\
\hline
\end{tabular}


Table S2: IFD simulations of 5-HT in hSERT with $\mathrm{Cl}^{-}$in the proposed site. Setup numbers correspond to those in Table 2.

\begin{tabular}{c|ccccccccc} 
Setup & $\begin{array}{c}\text { Homology } \\
\text { model }\end{array}$ & Sodium & $\begin{array}{c}\text { Binding } \\
\text { Site }\end{array}$ & $\begin{array}{c}\text { Scoring } \\
\text { Function }\end{array}$ & Poses & Cluster1 & Cluster2 & Cluster3 & Outlier \\
\hline 4-Cl & A-4 & Na1, Na2 & D98, I172 & SP & 6 & 1 & 5 & & \\
$6-C l$ & A-4 & Na1, Na2 & D98, I172 & XP & 6 & 1 & 5 & & \\
$15-C l$ & C-5 & Na1, Na2 & D98, I172 & SP & 8 & 6 & 1 & 1 & \\
$18-C l$ & C-5 & Na1, Na2 & D98, I172 & XP & 8 & 6 & 1 & 1
\end{tabular}

Table S3: Induced fit docking of 5-HT in homology models A-4 and C-5 of hSERT with $\mathrm{Cl}^{-}$in the proposed binding site. The setup number in column 1 refers to Table 2 in the manuscript. The poses are sorted with respect to cluster, IFD-score, and GlideScore.

\begin{tabular}{|c|c|c|c|c|c|c|c|}
\hline Setup & \begin{tabular}{|c} 
Prime E \\
(kcal/mol)
\end{tabular} & $\begin{array}{l}\text { IFDScore } \\
\text { (kcal/mol) }\end{array}$ & $\begin{array}{c}\text { GlideScore } \\
\text { (kcal/mol) }\end{array}$ & $\begin{array}{c}\text { E-Model } \\
\text { (kcal/mol) }\end{array}$ & Cluster & $\begin{array}{c}\text { Dist 5-HT(N)... } \\
\text { D98(O1) }(\AA)\end{array}$ & $\begin{array}{c}\text { Dist 5-HT(N) )... } \\
\text { D98(O2) }(\AA)\end{array}$ \\
\hline $15-\mathrm{Cl}$ & $\begin{array}{l}-19127 \\
\end{array}$ & -966.84 & -10.49 & -85.22 & $\mathrm{C} 1$ & 3.0 & 3.9 \\
\hline $15-\mathrm{Cl}$ & -19135 & -966.72 & -9.99 & -85.76 & $\mathrm{C} 1$ & 3.6 & 4.4 \\
\hline $15-\mathrm{Cl}$ & -19134 & -966.50 & -9.78 & -83.69 & $\mathrm{C} 1$ & 3.1 & 4.2 \\
\hline $18-\mathrm{Cl}$ & -19135 & -966.36 & -9.66 & -69.56 & C1 & 2.7 & 3.5 \\
\hline $15-\mathrm{Cl}$ & -19123 & -966.28 & -10.10 & -84.41 & C1 & 4.4 & 3.5 \\
\hline $18-\mathrm{Cl}$ & -19134 & -965.91 & -9.19 & -57.51 & $\mathrm{C} 1$ & 2.5 & 3.7 \\
\hline $15-\mathrm{Cl}$ & -19118 & -965.75 & -9.87 & -84.30 & $\mathrm{C} 1$ & 4.0 & 3.8 \\
\hline $15-\mathrm{Cl}$ & -19125 & -965.45 & -9.22 & -76.99 & $\mathrm{C} 1$ & 3.3 & 4.4 \\
\hline $18-\mathrm{Cl}$ & -19125 & -964.46 & -8.22 & -56.16 & C1 & 2.6 & 3.6 \\
\hline $18-\mathrm{Cl}$ & -19127 & -963.00 & -6.64 & -60.82 & $\mathrm{C} 1$ & 3.1 & 4.9 \\
\hline $18-\mathrm{Cl}$ & -19123 & -962.56 & -6.39 & -59.96 & $\mathrm{C} 1$ & 3.6 & 2.6 \\
\hline $18-\mathrm{Cl}$ & -10118 & -961.89 & -6.00 & -59.98 & C1 & 3.1 & 3.0 \\
\hline $4-\mathrm{Cl}$ & -18696 & -959.00 & -10.53 & -80.32 & $\mathrm{C} 1$ & 3.2 & 2.7 \\
\hline $6-\mathrm{Cl}$ & -18969 & -958.57 & -10.10 & -55.05 & $\mathrm{C} 1$ & 3.2 & 2.8 \\
\hline $15-\mathrm{Cl}$ & -19134 & -966.88 & -10.18 & -80.00 & $\mathrm{C} 2$ & 2.8 & 3.8 \\
\hline $18-\mathrm{Cl}$ & -19134 & -963.24 & -6.53 & -55.83 & $\mathrm{C} 2$ & 2.9 & 3.2 \\
\hline $4-\mathrm{Cl}$ & -18993 & -959.37 & -9.74 & -78.32 & $\mathrm{C} 2$ & 3.5 & 2.7 \\
\hline $4-\mathrm{Cl}$ & -18989 & -959.35 & -9.88 & -79.38 & $\mathrm{C} 2$ & 3.7 & 2.9 \\
\hline $4-\mathrm{Cl}$ & -18986 & -959.16 & -9.86 & -79.00 & $\mathrm{C} 2$ & 3.4 & 2.7 \\
\hline $4-\mathrm{Cl}$ & -18969 & -957.69 & -9.24 & -74.48 & $\mathrm{C} 2$ & 3.4 & 2.6 \\
\hline $4-\mathrm{Cl}$ & -18967 & -957.46 & -9.13 & -79.97 & $\mathrm{C} 2$ & 4.6 & 2.7 \\
\hline $6-\mathrm{Cl}$ & -18993 & -956.46 & -6.83 & -52.36 & $\mathrm{C} 2$ & 3.2 & 3.1 \\
\hline $6-\mathrm{Cl}$ & -18989 & -956.35 & -6.87 & -53.70 & $\mathrm{C} 2$ & 3.2 & 2.8 \\
\hline $6-\mathrm{Cl}$ & -18967 & -956.03 & -7.70 & -55.83 & $\mathrm{C} 2$ & 5.4 & 4.1 \\
\hline $6-\mathrm{Cl}$ & -18986 & -955.87 & -6.57 & -53.26 & C2 & 3.4 & 2.9 \\
\hline $6-\mathrm{Cl}$ & -18969 & -955.04 & -6.59 & -56.49 & $\mathrm{C} 2$ & 3.4 & 2.6 \\
\hline $18-\mathrm{Cl}$ & -19146 & -967.93 & -10.65 & -63.83 & C3 & 3.8 & 3.0 \\
\hline $15-\mathrm{Cl}$ & -19146 & -967.34 & -10.05 & -85.38 & C3 & 4.6 & 4.0 \\
\hline
\end{tabular}




\section{References}

(1) Yung-Chi, C.; Prusoff, W. H. Biochem. Pharmacol. 1973, 22, 3099-3108.

(2) Yamada, F.; Kobayashi, K.; Shimizu, A.; Aoki, N. Heterocycle 1993, 36, 2783-2804.

(3) Gower, B. G.; Leete, E. J. Am. Chem. Soc. 1963, 85, 3683-3685.

(4) Liang, G. B.; Desper, J. M.; Gellman, S. H. J. Am. Chem. Soc. 1993, 115, 925-938.

(5) Fish, M. S.; Johnson, N. M.; Horning, E. C. J. Am. Chem. Soc. 1956, 78, 3668-3671.

(6) Coyne, W. E.; Cusic, J. W. J. Med. Chem. 1968, 11, 1158-1160.

(7) Jackson, R. W.; Manske, R. H. J. Am. Chem. Soc. 1930, 52, 5029-5035.

(8) Pedersen, C. M.; Bols, M. Tetrahedron 2005, 61, 115-122.

(9) Eiter, K.; Svierak, O. L. Monatsh. Chem. 1952, 83, 1453-1476.

(10) Szmuszkovicz, J. J. Am. Chem. Soc. 1960, 82, 1180-1186. 